\title{
AVALIAÇÃO DO EFEITO DO AMBIENTE DE LOJA ON-LINE NAS INTENÇÕES DE APROXIMAÇÃO E REJEIÇÃO DOS CONSUMIDORES: UMA ANÁLISE POR MEIO DO PARADIGMA ESTÍMULO-ORGANISMO-RESPOSTA EVALUATION OF THE EFFECT OF THE ENVIRONMENT ON INTENT ONLINE STORE APPROACH AND CONSUMER REJECTION: AN ANALYSIS BY MEANS STIMULUS-ORGANISM-RESPONSE PARADIGM
}

\section{Evandro Luiz Lopes}

Professor doutor de Marketing do Programa de Pós-Graduação em Administração e de Métodos Quantitativos do Mestrado Profissional em Administração (Gestão de Sistemas) da Universidade Nove de Julho (Uninove), São Paulo (SP). Professor adjunto de Administração

Data de recebimento: 06-07-2015 Data de aceite: 09-09-2015 de Marketing e Comportamento do Consumidor e do Comprador Organizacional da Escola Paulista de Política, Economia e Negócios da Universidade Federal de São Paulo (Unifesp), São Paulo (SP), Brasil

\section{Eliane Herrero Lopes}

Mestranda em Administração pelo Centro Universitário das Faculdades Metropolitanas Unidas, FMU, Brasil

\section{RESUMO}

O propósito deste estudo é analisar a influência do ambiente de lojas online nas intenções de aproximação e rejeição dos consumidores, por meio do paradigma Estímulo-Organismo-Resposta. Com os dados de um survey, que coletou informações de 1.437 consumidores de um varejista virtual, analisados por meio de equações estruturais com estimação dos mínimos quadrados parciais, testamos as relações de um modelo conceitual previamente concebido. Os resultados obtidos identificaram a importância relativa das dimensões do ambiente de loja virtual na formação das emoções, positivas e negativas, e o efeito destas nas intenções comportamentais da amostra.

Palavras-chave: Ambiente de loja; loja on-line; paradigma Estímulo-Organismo-Resposta.

\section{ABSTRACT}

The purpose of this study is analyzing the influence of the online shopping environment on consumers' intentions of approximation and rejection through the paradigm Stimulus-Organism-Response. With data from a survey, which collected information from 1,437 consumers from a virtual retailer, analyzed using structural equations with partial minimal squares estimation, we tested the relations of a previously conceived conceptual model. The results show the relative importance of the dimensions of the virtual store environment in the formation of positive and negative emotions, and the effect of these on the sample's behavioral intentions.

Keywords: Store environment; online store; stimulus-organism-response paradigm.

Endereço dos autores: 


\section{INTRODUÇÃO}

Empresas totalmente voltadas à prestação de serviços já fazem parte de sociedade moderna há algum tempo. Contudo, o aumento da concorrência entre os prestadores de serviços tem forçado as organizações a ter mais atenção na natureza das intenções comportamentais dos clientes. Além disso, o crescimento do uso da web para aquisição de produtos e serviços, mudou o cenário varejista, criando uma nova modalidade de comercialização denominada e-tailing (KOERNIG, 2003).

Em um abrangente levantamento realizado pelo WordPress.com, identificou-se que houve grande crescimento do número de websites em operação, passando de 50 milhões em 2004 para mais de 155 milhões em 2008. Este crescimento expressivo é um dos resultados da grande atração que este novo ambiente de compras exerce sobre os consumidores (AUGER, 2005). Diante desta forte concorrência virtual, entende-se que atrair e reter consumidores no ambiente online é tão importante quanto em uma loja física, tornando este tema muito relevante, tanto para gestores quanto para acadêmicos.

Os atributos dos produtos e do ambiente de compras são constantemente utilizados pelos consumidores para avaliar a qualidade geral do ambiente varejista e contribuem para a elaboração de uma avaliação geral da imagem da loja (YUN \& GOOD, 2007). Imagem da loja, portanto, reflete a completa experiência que os clientes têm com os produtos e o ambiente de loja. Para os consumidores, a satisfação com as compras será um resultado direto das avaliações do ambiente e do produto que eles consideram importante (SHERMAN et al., 1997; SULLIVAN, 2002). Estudos anteriores identificaram que a imagem da loja tem influência direta sobre o comportamento positivo de aproximação e também na redução da evasão (SULLIVAN, 2002; EROGLU, MACHLEIT, \& DAVIS, 2003).
Estudos anteriores investigaram a relação entre a percepção do ambiente varejista e o comportamento de compra (BAKER et al., 2002; EROGLU, MACHLEIT, \& DAVIS, 2003; BRANDÃO, \& PARENTE, 2012) dando grande ênfase à percepção dos consumidores acerca de ambientes de lojas físicas (SWANSON \& DAVIS, 2003; BABIN et al., 2005). Como o projeto do website pode influenciar a atração e retenção de consumidores, a discussão sobre o ambiente de loja virtual também tem despertado o interesse da academia (WANG et al., 2002; CYR et al., 2005). Acreditase que uma contribuição é analisá-lo por meio do paradigma Estímulo-Organismo-Resposta (originalmente, Stimulus-Organism-Reaction [S-O-R]) proposto por Mehrabian e Russell (1974).

Algumas iniciativas de pesquisa proporcionaram uma visão inicial sobre a aplicação do paradigma Estímulo-Organismo-Resposta no ambiente online, no entanto, tais estudos forneceram apenas fundamentos conceituais, sem evidências empíricas, sugerindo o estímulo ambiental global como única variável antecedente da intenção comportamental (BOSMANS, 2006). Além disso, existe pouco conhecimento sobre a relação entre o design do website e o comportamento de compras online (SONG \& ZAHADI, 2005).

A fim de preencher essa lacuna da literatura, este trabalho está estruturado em quatro seções, além desta introdução. A primeira seção apresentará a revisão da literatura que possibilitou a geração das hipóteses do trabalho. A seção seguinte apresentará os procedimentos metodológicos utilizados na fase empírica. A terceira seção apresentará os resultados obtidos no campo e, finalmente, a última seção apresentará a discussão dos resultados e os comentários finais.

\section{REVISÃO DA LITERATURA}

Nesta seção será apresentado o arcabouço teórico que alicerçou esta pesquisa. 


\subsection{O paradigma Estímulo-Organismo- Resposta}

O paradigma Estímulo-Organismo-Resposta [E-O-R] (MEHRABIAN \& RUSSELL, 1974) sugere que estímulos ambientais influenciam fortemente o comportamento de aproximação (uso) e evasão (troca). O paradigma é delineado por três componentes sequenciais, sendo estes os estímulos ambientais percebidos (Estímulo), a perspectiva emocional e cognitiva dos consumidores (Organismo) e os resultados de aproximação/evasão do consumidor (Resposta).

O paradigma E-O-R vem sendo utilizado nas investigações dos estímulos ambientais no varejo físico e na influência desses estímulos no comportamento do consumidor. Os resultados geralmente suportam a aplicabilidade do paradigma, identificando que os estímulos ambientais varejistas influenciam o comportamento do consumidor de forma significativa (BAKER et al., 1992; SHERMAN et al., 1997; SULLIVAN, 2002; BRANDÃO \& PARENTE, 2012).

Poucos trabalhos têm utilizado o paradigma E-O-R para analisar o comportamento do consumidor na web. Além disso, esses estudos adotaram a perspectiva integrada dos estímulos ambientais, sem que haja a possibilidade de identificar o efeito de cada grupo de atributos na intenção ou comportamento dos consumidores. A Figura 1 apresenta as principais iniciativas neste sentido.

\begin{tabular}{|c|c|c|}
\hline Autor(es) & Periódico & Principais achados \\
\hline Eroglu et al. (2001) & Journal of Business Research & $\begin{array}{l}\text { O paradigma Estímulo-Organismo-Resposta foi utilizado como } \\
\text { a base do modelo que postula que as característica ambientais } \\
\text { de uma loja online influenciam os estados afetivos e cognitivos } \\
\text { dos consumidores e influenciam os comportamentos de } \\
\text { aproximação e evasão. Identificaram ainda que o envolvimento } \\
\text { e capacidade de resposta atmosférica do consumidor moderam } \\
\text { a relação entre os estímulos ambientais e reações afetivas e } \\
\text { cognitivas dos compradores. }\end{array}$ \\
\hline $\begin{array}{l}\text { Groeppel-Klein e Baun } \\
\qquad(2001)\end{array}$ & Advances in Consumer Research & $\begin{array}{l}\text { Os autores verificaram a influência do ambiente da loja influencia } \\
\text { a excitação dos consumidores, mensurada por meio da variância } \\
\text { eletrodérmica da pele, comparando dois ambientes varejistas } \\
\text { distintos. }\end{array}$ \\
\hline Wang et al. (2002) & $\begin{array}{c}\text { The Proceedings of American } \\
\text { Marketing Association }\end{array}$ & $\begin{array}{l}\text { Identificaram que o ambiente da loja online modera o risco } \\
\text { percebido com a transação eletrônica. }\end{array}$ \\
\hline Costa e Larán (2003) & Revista de Administração de Empresas & $\begin{array}{l}\text { Identificaram a influência do ambiente da loja na impulsividade } \\
\text { do consumidor. }\end{array}$ \\
\hline McKinney (2004) & $\begin{array}{l}\text { International Journal of Consumer } \\
\text { Studies }\end{array}$ & $\begin{array}{l}\text { Identificou que a influência da orientação do consumidor - } \\
\text { para compras físicas ou virtuais - na satisfação é parcialmente } \\
\text { anulada pela avaliação do ambiente de loja. }\end{array}$ \\
\hline Mummalaneni (2005) & Journal of Business Research & $\begin{array}{l}\text { Analisando o ambiente da loja de maneira integrada, o } \\
\text { estudo propõe a estrutura Estímulo-Organismo-Resposta } \\
\text { como um modelo viável para a investigação das respostas dos } \\
\text { consumidores para os websites de lojas virtuais. }\end{array}$ \\
\hline
\end{tabular}

Figura 1 - Principais trabalhos publicados sobre ambiente de lojas virtuais 


\subsection{Ambiente de lojas físicas versus virtuais}

O ambiente de lojas físicas atrai grande interesse dos acadêmicos que centram suas pesquisas na avaliação do impacto dessas sobre o comportamento do consumidor (GARDNER \& SIOMKOS, 1986; TURLEY \& MILLIMAN, 2000).

Assim como varejistas tradicionais transmitem informações acerca de sua imagem e estratégias de negócio por meio do ambiente que implementam em suas lojas, os varejistas online utilizam várias ferramentas para proporcionar aos internautas a melhor experiência de compra possível.

Ambiente varejista virtual pode ser definido como a concepção consciente da web para criar efeitos positivos nos usuários (EROGLU, MACHLEIT, \& DAVIS, 2003), a fim de aumentar a resposta favorável do consumidor (DAILEY, 2004).

Para lojas físicas, Lewison (1994) desenvolveu um quadro teórico que suporta a tipificação do ambiente de loja de varejo em três componentes básicos: imagem da loja, efeitos "atmosféricos" e "teatralidade" do ambiente. No entanto, a classificação dos atributos ambientes das lojas tradicionais não podem ser transpostos integralmente para a análise dos atributos ambientais das lojas virtuais (EROGLU, MACHLEIT, \& DAVIS, 2003).

Baseando-se no trabalho de Lewison (1994), Vrechopoulos et al.(2004) adaptou os componentes do ambiente de varejo convencional ao cenário virtual, classificando-os também em quatro dimensões: layout virtual e design, atmosfera virtual, teatralidade virtual e presença social virtual. Esse quarto componente, que a primeira vista causa certa estranheza, visto que a presença física e interação entre os vendedores e compradores não seja diretamente aplicável, é percebido por meio de chats e disponibilização de espaços para comentários de visitantes (EROGLU et al., 2001).

Com base nos princípios da psicologia ambiental, o paradigma E-O-R sugere que os estados emocionais dos consumidores moderam a relação entre o estímulo ambiental e as respostas dos consumidores. Sob este aspecto, Mummalaneni (2005 ) propõe a estrutura E-O-R como um modelo viável para a investigação do comportamento do consumidor online, pois a dimensão Organismo do paradigma encarna estados afetivos e cognitivos dos consumidores, resultando nos comportamentos de aproximação ou evasão.

\subsection{Resultados de pesquisas empíricas sobre o ambiente de loja online}

Nesta seção apresentaremos os resultados empíricos de pesquisas que estudaram o ambiente de lojas online e seus reflexos no comportamento de consumo.

\subsubsection{Layout e design virtual}

Basicamente, os layouts dos websites podem ser classificados em fixo, líquido ou elástico. O layout Líquido permite que a página se adapte à largura da tela do usuário, ocupando todo o espaço visível em vez de ficar encostada a um canto ou centralizada, como acontece com os layouts fixos. Sua principal vantagem é a acessibilidade (ou usabilidade). O layout, assim como seu conteúdo, adapta-se automaticamente à qualquer resolução de tela utilizada pelo usuário, ocupando toda área visível, ou seja, não restam espaços vazios nas laterais. O principal problema deste tipo de layout é que se perde o controle sobre o posicionamento de alguns elementos da página, fazendo com que o mesmo possa ficar desconfigurado, com elementos deslocados e com linhas de texto muito longas.

O layout fixo é o mais utilizado pela grande maioria dos websites, isso por que as páginas terão sempre a mesma largura e os conteúdos irão comportar-se sempre da mesma maneira. Este modelo de layout tem como principal desvantagem a falta de flexibilidade. Quando o website é 
adaptado para monitores de resolução baixa, muito espaço, sem utilização, fica aparente na tela. Por outro lado, se o website for construído para monitores widescreen, mas for acessado em monitores menores, será necessário usar a barra de rolagem para visualizá-lo.

A terceira opção, não muito comum, é o chamado layout elástico ou "adaptativo". Nesta situação, o website é autoajustável, dependendo da configuração do monitor que o está acessando. O programador pode definir a largura mínima e a máxima, o que permite um maior controle de imagens e textos e ao mesmo tempo controle da largura da janela por parte do usuário, eliminando assim alguns pontos negativos identificados nos dois tipos de layouts anteriores. Seu uso não é muito frequente, pois o tempo necessário para criar um website com este layout é muito maior que para os demais, sendo necessário testá-lo manualmente em todos os dispositivos possível. Com a frequência de lançamentos de novos hardwares, torna-se muito difícil manter controle da resolução da tela de todos os dispositivos existentes.

O layout da loja online define, em grande medida, a navegação dos consumidores dentro do website. Há indícios de que os consumidores percebam os layouts fixos como sendo os mais adequados e úteis na busca dos produtos desejados (VRECHOPOULOs et al., 2004). Da mesma forma, um layout fixo em uma loja online é percebido como mais fácil de usar e estimula respostas mais positivas do consumidor (GRIFFITH , 2005).

Diante disso, espera-se:

H1a - Haverá relação positiva entre o layout da loja online e as emoções positivas do consumidor em relação ao varejista virtual.

H1a - Haverá relação negativa entre o layout da loja online e as emoções negativas do consumidor em relação ao varejista virtual.

\subsubsection{Atmosfera virtual}

A cor é um elemento atmosférico que é presente na esmagadora maioria dos websites e o entendimento de sua influência atrai a atenção de muitos pesquisadores e gestores.

O efeito da cor na resposta comportamental dos consumidores já foi bastante testado, apresentando resultados estáveis (WU E YUAN, 2003).

Gorn et al. (2004) descobriram que as cores frias, por induzem a estados de sentimento mais relaxado resultam na maior rapidez percebida nas situações de download. A cor também afeta a avaliação dos usuários resultando na maior probabilidade de recomendação do website (GORN et al., 2004) e na avaliação da personalidade da loja (CLARK et al., 2004).

Em estudos anteriores, identificou-se que quando um item de valor agregado alto foi apresentado em uma cor de fundo "quente", houve maior intenção de compra declarada pelos usuários (BIERS \& RICHARDS, 2005). Identificaram também que a combinação de certas cores afeta a preferência visual e o desempenho de leitura dos consumidores (WU \& YUAN, 2003).

Apesar dos diversos estudos realizados sobre os efeitos da música em lojas convencionais, não se localizou nenhum estudo empírico que investigou seu efeito sobre as respostas dos consumidores online. Sob esta ótica, espera-se encontrar o seguinte relacionamento:

H2a - Haverá relação positiva entre a Atmosfera virtual da loja online e as emoções positivas do consumidor em relação ao varejista virtual.

H2b - Haverá relação negativa entre a Atmosfera virtual da loja online e as emoções negativas do consumidor em relação ao varejista virtual. 


\subsubsection{Teatralidade virtual}

Varejistas virtuais demonstram grande habilidade em capturar a atenção dos consumidores online por meio do uso de imagens, gráficos, animações e ícones. Em um experimento conduzido com 117 estudantes universitários, Martin et al.et al (2005) identificaram que websites de média complexidade são mais bem avaliados que os de alta ou baixa complexidade, termo que se deve à riqueza de elementos - imagens, gráficos e animações - utilizados na configuração da página (ROSEN \& PURINTON , 2004). Estes resultados confirmaram os achados de Lee e Benbasat (2003), que identificaram atitudes menos positivas na avaliação de websites de alta complexidade, talvez porque estes exigem maior atenção do usuário, podendo resultar em cansaço.

A interatividade, outro elemento da teatralidade virtual, é expressa pela possibilidade de participação do usuário na construção ou modificação, em tempo real, das páginas do website (FIORE et al., 2005). Ambientes virtuais mais interativos resultam em avaliações mais positivas quando comparados a websites menos interativos (COYLE \& THORSON, 2001). Contudo, há indícios que afirmam que o aumento da nitidez da mensagem, por meio de cores, gráficos e animação é mais efetivo na geração sentimentos favoráveis do que altos níveis de interatividade (DHOLAKIA, 2005). Com isto:

H3a - Haverá relação positiva entre a Teatralidade da loja online e as emoções positivas do consumidor em relação ao varejista virtual.

H3b - Haverá relação negativa entre a Teatralidade da loja online e as emoções negativas do consumidor em relação ao varejista virtual.

\subsubsection{Presença social virtual}

Os efeitos da presença social virtual sobre as respostas dos consumidores online não estão bem documentados na literatura. Mesmo que os websites disponibilizem ferramentas para que os consumidores comuniquem suas preferências aos demais visitantes, ainda não está claro qual o efeito gerado. Em um dos parcos estudos realizados sobre a temática, Clark et al. (2004) não encontrou efeitos em relação à presença de um avatar ou persona virtual sobre a personalidade percebida da loja virtual. Contudo, não se verificou empiricamente a relação entre a presença social virtual e a atitude em relação ao varejista. Para fins de verificação do efeito desta dimensão, propomos testar:

H4a - Haverá relação positiva entre a Presença social virtual na loja online e as emoções positivas do consumidor em relação ao varejista virtual.

H4b - Haverá relação negativa entre a Presença social virtual na loja online e as emoções negativas do consumidor em relação ao varejista virtual.

Ainda considerando o paradigma EstímuloOrganismo-Resultado, podemos sugerir:

H5a - Haverá relação positiva entre as emoções positivas do consumidor online e a intenção comportamental de aproximação ao varejista.

H5b - Haverá relação negativa entre as emoções positivas do consumidor online e a intenção comportamental de rejeição ao varejista.

H5c - Haverá relação positiva entre as emoções negativas do consumidor online e a intenção comportamental de rejeição ao varejista.

H5d - Haverá relação negativa entre as emoções negativas do consumidor online e a intenção comportamental de aproximação ao varejista.

Com o objetivo de facilitar o entendimento do framework deste estudo, elaboramos a Figura 2. 


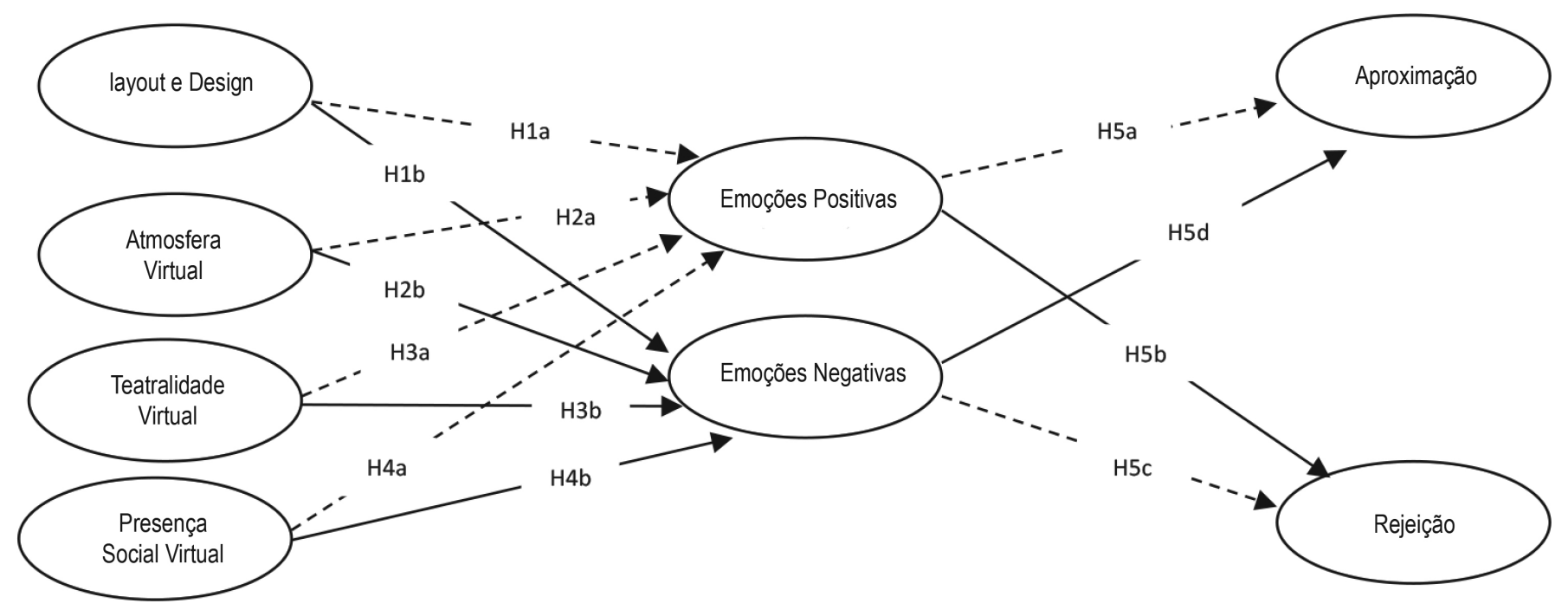

Figura 2 - Modelo conceitual do estudo

Setas tracejadas indicam relações positivas enquanto as setas contínuas indicam relações negativas.

\section{MÉTODO}

O objetivo desta seção é descrever o método utilizado na condução da fase empírica deste estudo.

\subsection{Plano de pesquisa}

Visando responder à questão de pesquisa proposta, planejamos realizar a etapa empírica do projeto em duas fases, sendo a primeira exploratória e a segunda descritiva.

\subsection{Fase exploratória}

Inicialmente, realizamos um focus group, com o objetivo de conhecer os atributos ambientais dos varejistas online que mais influenciavam o processo de decisão de compra dos consumidores. Os participantes foram convidados por meio de um sorteio aleatório realizado no banco de dados de um varejista online. Contamos com a participação de sete consumidores na maioria mulheres ( $n=4 ; 57 \%$ ), com idade média de 32,9 anos (mínimo=24; $d p=8,1$ ), que foram convidados com antecedência para a realização da tarefa. Ofereceu-se um brinde para cada participante, como forma de estimular o comparecimento no dia e local previamente combinados.

A sessão foi realizada em uma sala preparada para esta finalidade sendo que um dos autores atuou como facilitador da reunião. Os participantes listaram livremente, durante quarenta minutos, todos os atributos de uma loja online que seriam relevantes para ajudá-los no processo de decisão de compras. Os atributos considerados, em sua grande maioria, foram resultado do consenso do grupo, porém, nas raras divergências, a seleção do atributo ocorreu pela votação da maioria dos participantes, após discussão sobre a sua importância para o processo decisório.

A atividade resultou em uma lista de 21 atributos varejistas

Com utilização do software Atlasti, os autores categorizaram os atributos listados durante 0 grupo focal, classificando-os nas quatro dimensões do ambiente de loja virtual preconizadas por Vrechopoulos et al.(2004).

Desprezamos os itens que se referiam a preços, promoções, políticas de troca e pontualidade de entrega (entre outros) por não serem o foco 
deste estudo. O resultado da categorização, que pode ser visto na Figura 3, agrupou nove itens na dimensão layout e design virtual (Lay), quatro itens na dimensão Atmosfera virtual (Av), cinco itens na dimensão Teatralidade virtual (Tv) e, por fim, três itens na dimensão Presença social virtual (Psv).

Para a validação de conteúdo (DEVELLIS, 2011) a classificação realizada pelos autores foi

\begin{tabular}{|c|c|c|}
\hline Dimensão & Código - Atributos & $\begin{array}{l}\text { Validação de } \\
\text { Conteúdo }\end{array}$ \\
\hline \multirow{9}{*}{$\begin{array}{l}\text { Layout e design virtual } \\
\text { (Lay) }\end{array}$} & Lay01 - Apresentação estética geral do website & $100 \%$ \\
\hline & Lay02 - Facilidade de navegação dentro do website & $100 \%$ \\
\hline & Lay03 - Disposição das barras de ferramentas & $83 \%$ \\
\hline & Lay04 - Facilidade de visualização & $100 \%$ \\
\hline & Lay05 - Aparência moderna da webpage & $100 \%$ \\
\hline & Lay06 - Informações claras e objetivas & $100 \%$ \\
\hline & Lay07 - Departamentalização dos produtos & $100 \%$ \\
\hline & Lay08 - Facilidade de pesquisa de produtos & $100 \%$ \\
\hline & Lay09 - Avaliações de qualidade dos produtos & $83 \%$ \\
\hline \multirow{4}{*}{$\begin{array}{l}\text { Atmosfera virtual } \\
\text { (Av) }\end{array}$} & Av01 - Cores das letras que facilitem o entendimento & $100 \%$ \\
\hline & Av02 - Cores do website que "não forcem a vista" & $100 \%$ \\
\hline & Av03 - Harmonia entre informações e produtos & $100 \%$ \\
\hline & Av04 - Qualidade sonora do website & $100 \%$ \\
\hline \multirow{5}{*}{$\begin{array}{c}\text { Teatralidade virtual } \\
\text { (Tv) }\end{array}$} & Tv01 - Qualidade das imagens & $100 \%$ \\
\hline & Tv02 - Qualidade das fotos & $100 \%$ \\
\hline & Tv03 - Possibilidade de desabilitar poup-ops & $100 \%$ \\
\hline & Tv04 - Ícones estáticos e de fácil operação & $100 \%$ \\
\hline & Tv05 - Possibilidade de personalizar a webpage inicial & $100 \%$ \\
\hline \multirow{3}{*}{$\begin{array}{l}\text { Presença social virtual } \\
\text { (Psv) }\end{array}$} & Psv01 - Chat disponível e operacional & $100 \%$ \\
\hline & Psv02 - Espaço para indicar a qualidade dos produtos comprados & $83 \%$ \\
\hline & Psv03 - Imagem do atendente do Chat & $100 \%$ \\
\hline
\end{tabular}

Figura 3 - Atributos listados na fase exploratória

submetida, em ordem aleatória, à avaliação de seis professores doutores em Administração com linhas de pesquisa em Marketing, para que indicassem a qual dimensão pertenciam. A validação de conteúdo foi satisfatória, pois houve assimetria no entendimento de apenas três itens por um dos avaliadores, resultando em $83 \%$ de conformidade, superando assim os $80 \%$ necessários (MALHOTRA, 2006).

\subsection{Fase descritiva}

Após finalizada a etapa exploratória que gerou a lista de atributos, partimos para a realização de um survey em corte transversal, como descrito a seguir.

\subsection{Escalas e instrumento de coleta de dados}

Para mensuração do Ambiente de Loja online, utilizamos os 21 itens gerados na fase exploratória. 0 respondente deveria avaliar cada um dos itens, apresentados em ordem aleatória, por meio de uma escala de 10 pontos ancorados em 1- Péssimo(a) e 10-Ótimo(a). A avaliação deveria refletir a opinião do respondente sobre a Loja online na qual tivesse realizado o maior número de transações nos seis meses anteriores à pesquisa.

Para mensuração das emoções positivas e negativas, utilizamos os 12 itens da escala Consumption Emotions Set (RICHINS, 1997), avaliados por meio de uma escala de 10 pontos ancorados em 1- nem um pouco e 10- extremamente. A escala de Richins (1997) já foi amplamente validade para avaliações das emoções 
em situações de consumo, sendo formada por quatro itens para avaliação de emoções positivas (alegre, feliz, contente, animado[a]) e oito itens para mensuração das emoções negativas (frustrado[a], deprimido[a], com raiva, irritado[a], furioso[a], triste, infeliz, desamparado[a]).

Finalmente, para a mensuração dos construtos Aproximação e Rejeição, utilizamos uma escala de oito afirmativas, sendo quatro para cada dimensão, adaptadas de Hardesty, Carlson e Bearden (2002) e de Hsiao, Sun e Morwitz (2002) respectivamente. Todos os itens foram mensurados por meio de uma escala de 10 pontos ancorados em 1- Discordo Totalmente e 10Concordo Totalmente e são apresentados na Figura 4.

\begin{tabular}{|c|c|c|}
\hline Código & Afirmativa & Fonte \\
\hline AP01 & $\begin{array}{c}\text { Continuarei comprando desta } \\
\text { loja virtual. }\end{array}$ & \multirow{4}{*}{$\begin{array}{c}\text { Hardesty, } \\
\text { Carlson e } \\
\text { Bearden } \\
(2002)\end{array}$} \\
\hline AP02 & $\begin{array}{c}\text { Certamente recomendarei esta } \\
\text { loja virtual para meus parentes e } \\
\text { amigos. }\end{array}$ & \\
\hline AP03 & $\begin{array}{c}\text { Comprar nesta loja virtual é uma } \\
\text { excelente escolha. }\end{array}$ & \\
\hline AP04 & $\begin{array}{c}\text { Tenho grande confiança nesta } \\
\text { loja virtual. }\end{array}$ & \\
\hline RJ01 & $\begin{array}{l}\text { No futuro tentarei fazer minhas } \\
\text { compras em outra loja virtual. }\end{array}$ & \multirow{4}{*}{$\begin{array}{l}\text { Hsiao, Sun } \\
\text { e Morwitz } \\
\quad(2002)\end{array}$} \\
\hline RJ02 & $\begin{array}{c}\text { Sempre que posso, pesquiso } \\
\text { produtos em outras lojas } \\
\text { virtuais. }\end{array}$ & \\
\hline RJ03 & $\begin{array}{c}\text { Compro nesta loja virtual } \\
\text { somente porque não tenho } \\
\text { outra opção melhor. }\end{array}$ & \\
\hline RJ04 & $\begin{array}{c}\text { Quando encontrar outra loja } \\
\text { virtual não comprarei mais } \\
\text { desta. }\end{array}$ & \\
\hline
\end{tabular}

Figura 4 - Variáveis de mensuração de aproximação e rejeição

O questionário foi submetido aos respondentes, em formato digital, por meio do website QuestionPro.

\subsection{Amostra e procedimentos de campo}

Após um pré-teste do questionário, no qual foram ajustados poucos detalhes na redação de alguns itens, iniciamos a coleta dos dados.

Uma grande empresa varejista enviou o link do formulário digital para 17.000 consumidores que haviam realizado compras em seu website nos últimos seis meses. Com isto, a amostra pode ser classificada como não probabilística por conveniência (MALHOTRA, 2006). Além do link, uma carta apresentando o caráter acadêmico da pesquisa foi enviada. Ademais, a empresa varejista disponibilizou um brinde para ser sorteado entre os respondentes do questionário. A coleta de dados aconteceu em Dezembro de 2013.

\subsection{Plano de análise de dados}

Para a análise dos relacionamentos entre os construtos, escolheu-se a técnica de modelagem de equações estruturais (MEE), com a análise prévia da colinearidade e a verificação da normalidade (HAIR et. al, 2005), para suportar a decisão entre a utilização da análise por meio da matriz de covariância (LISREL) e/ou da matriz de correção com estimação por meio de mínimos quadrados parciais (Partial Least Square - PLS) (CHIN, 1998).

\section{RESULTADOS}

O objetivo desta seção é apresentar os resultados da análise dos dados obtidos na fase empírica do estudo.

\subsection{Descrição da amostra}

O questionário foi acessado por 9.145 consumidores $(53,7 \%)$, porém somente $1.437(8,4 \%)$ internautas responderam a todas as questões, sendo estes considerados como a amostra final deste estudo.

A maioria dos respondentes é do sexo feminino ( $n=917 / 63,9 \%)$, com idade média de 28 anos $(d p=6,5)$. A maior parte finalizou o ensino médio ( $n=686 / 47,7 \%$ ) ou possui nível superior completo ( $n=329 / 22,8 \%$ ). A grande maioria exerce função 
remunerada ( $n=1.104 / 76,8 \%$ ) com salário superior à $\mathrm{R} \$ 2.500,00(n=614 / 42,7 \%)$.

Curiosamente, somente $54,3 \%$ dos pesquisados $(n=781)$ responderam o questionário com base no website mantido pelo varejista que realizou o convite para a pesquisa. Os demais fizeram suas avaliações em relação a outros websites, nos quais costumam realizar compras. A frequência de compras realizadas no último semestre foi relativamente elevada e está demonstrada na Tabela 1.

Tabela 1 - Frequência de compras declarada pela amostra

\begin{tabular}{lccc}
\hline $\begin{array}{c}\text { Frequência de compras } \\
\text { nos últimos } 6 \text { meses }\end{array}$ & $\mathbf{n}$ & $\%$ & $\begin{array}{c}\% \\
\text { acumulada }\end{array}$ \\
\hline 1 ou 2 compras & 425 & $29,6 \%$ & $29,6 \%$ \\
3 ou 4 compras & 321 & $22,3 \%$ & $51,9 \%$ \\
5 ou 6 compras & 237 & $16,5 \%$ & $68,4 \%$ \\
7 ou 8 compras & 199 & $13,8 \%$ & $82,3 \%$ \\
9 ou 10 compras & 82 & $5,7 \%$ & $88,0 \%$ \\
11 ou 12 compras & 111 & $7,7 \%$ & $95,7 \%$ \\
acima de 13 compras & 62 & $4,3 \%$ & $100,0 \%$ \\
Total & 1437 & $100,0 \%$ & \\
\hline
\end{tabular}

\subsection{Validação das escalas}

A validação das escalas foi obtida por meio da análise fatorial exploratória (AFE) com a utilização do SPSS 18.

A primeira tentativa de AFE da escala de emoções indicou a necessidade de eliminarmos dois itens, um por baixa comunalidade (desamparado) e outro por carga fatorial cruzada (animado). Após a eliminação desses itens, a AFE identificou dois fatores que explicaram 70,05\% da variância total. Além dos indicadores KMO $(0,827)$, esferacidade de Bartlett $\left(c^{2}{ }_{(45 \text { g...) }}=\right.$ 6904,09; $p<0,01 \%$ ) e os Alfas de Cronbach serem adequados, os itens se ajustaram às dimensões teoricamente corretas. O resultado desta análise pode ser visto na Tabela 2 .
Tabela 2 - AFE das dimensões emocionais

\begin{tabular}{cccc}
\hline Itens & $\begin{array}{c}\text { Emoções } \\
\text { Negativas }\end{array}$ & $\begin{array}{c}\text { Emoções } \\
\text { Positivas }\end{array}$ & Alpha \\
\hline alegre & $-0,011$ & 0,990 & \\
feliz & $-0,179$ & 0,936 & 0,914 \\
contente & $-0,189$ & 0,939 & \\
frustrado & 0,699 & $-0,201$ & \\
deprimido & 0,769 & $-0,114$ & \\
com_raiva & 0,796 & $-0,046$ & \\
irritado & 0,791 & $-0,070$ & 0,843 \\
furioso & 0,803 & $-0,144$ & \\
triste & 0,755 & $-0,148$ & \\
infeliz & 0,658 & $-0,268$ & \\
\% Variância Explicada & 45,04 & 25,01 & \\
\hline
\end{tabular}

Realizamos nova AFE para verificar a dimensionalidade dos construtos Rejeição e Aproximação. Logo na primeira tentativa, identificamos indicadores de ajustes adequados ( $\mathrm{KMO}=0,766$; Bartlett $=\left(c^{2}{ }_{(28 g . \text { I. })}=3547,42 ; p<0,01 \%\right)$ e consistência interna dentro dos limites aceitáveis). Identificamos, também, que os itens se ajustaram à composição fatorial teórica. O resultado da AFE pode ser visto na Tabela 3.

Tabela 3 - AFE das dimensões Rejeição e Aproximação

\begin{tabular}{cccc} 
Itens & Rejeição & Aproximação & Alpha \\
\hline AP01 &, 105 &, 726 & \\
AP02 &,- 358 &, 462 & 0,774 \\
AP03 &,- 182 &, 789 & \\
AP04 &,- 246 &, 755 & \\
RJ01 &, 682 &,- 361 & \\
RJ02 &, 726 &,- 309 & 0,789 \\
RJ03 &, 839 &, 015 & \\
RJ04 &, 797 &,- 020 & \\
\% Variância & 41,91 & 18,09 & \\
Explicada & & &
\end{tabular}

Na terceira e última AFE, verificamos a escala de Ambiente de Loja online desenvolvida neste trabalho. Na primeira tentativa, todos os itens da escala se ajustaram dentro da distribuição teórica. Contudo, a carga do item Psv03 foi negativa $(-0,570)$ e o Alfa de Cronbach da dimensão Personagem social virtual foi de 0,654. 
Mesmo que aceitável, com a remoção do item com carga negativa, o Alfa passou para 0,860. Com isto, decidimos eliminar a variável e realizamos nova AFE.
Nesta nova tentativa, identificamos quatro fatores que explicaram 56,83\% da variância da amostra e indicadores aceitáveis $\left(\mathrm{KMO}=0,847\right.$; Bartlett $=\mathrm{c}^{2}{ }_{(210 \mathrm{~g} . \mathrm{I.})}=$ 14232,15; $p<0,01 \%)$, como pode ser visto na Tabela 4.

Tabela 4 - AFE das dimensões do Ambiente de Loja online

\begin{tabular}{|c|c|c|c|c|c|}
\hline Itens & Atmosfera Virtual & Teatralidade virtual & Presença social virtual & Layout & Cronbach \\
\hline Lay01 & ,291 & ,058 & 111 &, 551 & \multirow{9}{*}{0,743} \\
\hline Lay02 &,- 065 & ,498 & ,329 & ,496 & \\
\hline Lay03 & , 186 & ,343 & 176 &, 512 & \\
\hline Lay04 &, 161 &, 004 & 200 &, 554 & \\
\hline Lay05 & 327 &,- 077 & 107 & 695 & \\
\hline Lay06 & 218 &,- 056 & ,347 &, 572 & \\
\hline Lay07 & , 186 & 072 & 021 & 741 & \\
\hline Lay08 & 373 &,- 072 & ,053 & ,650 & \\
\hline Lay09 & 324 &, 049 & ,088 &, 512 & \\
\hline Av01 &, 524 & ,067 & ,378 & 162 & \multirow{4}{*}{0,844} \\
\hline $\mathrm{Av02}$ &, 523 & ,047 & ,324 & ,341 & \\
\hline Av03 & ,615 &, 051 & 156 & 310 & \\
\hline $\mathrm{Av04}$ &, 618 & ,042 & 315 & 066 & \\
\hline Tv01 &, 042 & ,726 & 291 & 013 & \multirow{4}{*}{0,785} \\
\hline Tv02 & ,099 & ,777 & 091 &,- 018 & \\
\hline Tv03 & ,375 & ,676 &,- 088 & 267 & \\
\hline Tv04 &, 054 & ,742 & 141 & ,094 & \\
\hline Tv05 &, 065 & ,926 &,- 102 & 015 & \multirow{4}{*}{0,860} \\
\hline Psv01 & ,085 &,- 059 & 928 & 013 & \\
\hline Psv02 & ,030 &,- 085 & 867 & ,051 & \\
\hline ância Explicada & 28,92 & 15,1 & 6,68 & 6,11 & \\
\hline
\end{tabular}

Identificamos ausência de multicolinearidade, por meio do indicador de inflação da variância (VIF), indicando que a amostra é adequada para técnicas regressivas. Contudo, os indicadores KS de Kolmogorov-Smirnov apontam para a ausência de normalidade em todos os indicadores (Tabela 5). Com isto, optamos pela realização da modelagem de equações estruturais com estimação dos mínimos quadrados parciais e utilização do software SmartPLS2.0M3.

Tabela 5 - AFE das dimensões do Ambiente de Loja online

\begin{tabular}{ccccccccc}
\hline Itens & $\mathbf{n}$ & Média & Desvio Padrão & Mínimo & Máximo & VIF & KS & p-value \\
\hline Lay01 & 1437 & 9,03 & 1,340 & 1 & 10 & 1,664 & 9,725 &, 000 \\
Lay02 & 1437 & 7,90 & 2,807 & 1 & 10 & 3,416 & 10,272 &, 000 \\
Lay03 & 1437 & 7,59 & 3,107 & 1 & 10 & 3,282 & 9,646 &, 000 \\
Lay04 & 1437 & 7,79 & 2,227 & 1 & 10 & 1,608 & 6,142 &, 000 \\
Lay05 & 1437 & 8,96 & 1,270 & 1 & 10 & 1,975 & 8,916 &, 000 \\
Lay06 & 1437 & 8,68 & 1,705 & 1 & 10 & 1,886 & 8,342 \\
Lay07 & 1437 & 8,75 & 1,865 & 1 & 10 & 2,392 & 9,879 &, 000 \\
Lay08 & 1437 & 9,00 & 1,185 & 1 & 10 & 2,034 & 8,888 &, 000 \\
Lay09 & 1435 & 8,69 & 1,861 & 1 & 10 & 1,726 & 9,097 &, 000 \\
Av01 & 1437 & 8,80 & 1,404 & 1 & 10 & 1,840 & 8,135 &, 000 \\
\hline
\end{tabular}


Tabela 5 - Continuação

\begin{tabular}{ccccccccc}
\hline Itens & $\mathbf{n}$ & Média & Desvio Padrão & Mínimo & Máximo & VIF & KS & p-value \\
\hline Av02 & 1437 & 8,03 & 1,822 & 1 & 19 & 1,870 & 5,638 &, 000 \\
Av03 & 1437 & 8,10 & 2,087 & 1 & 10 & 2,134 & 7,448 &, 000 \\
Av04 & 1437 & 9,00 & 1,426 & 1 & 10 & 1,922 & 9,360 &, 000 \\
Tv01 & 1437 & 8,77 & 1,634 & 1 & 10 & 2,266 & 8,554 &, 000 \\
Tv02 & 1437 & 8,42 & 2,033 & 1 & 10 & 1,756 & 8,301 &, 000 \\
Tv03 & 1437 & 8,06 & 2,356 & 1 & 10 & 2,237 & 8,198 &, 000 \\
Tv04 & 1437 & 8,88 & 1,655 & 1 & 10 & 2,193 & 9,423 &, 000 \\
Tv05 & 1437 & 6,30 & 4,166 & 1 & 10 & 3,491 & 10,420 &, 000 \\
Psv01 & 1437 & 6,01 & 4,070 & 1 & 10 & 2,209 & 9,618 &, 000 \\
Psv02 & 1437 & 5,85 & 4,145 & 1 & 10 & 3,293 & 9,048 &, 000 \\
alegre & 1437 & 7,78 & 3,080 & 1 & 10 & 1,688 & 10,493 &, 000 \\
feliz & 1437 & 8,44 & 2,676 & 1 & 10 & 4,135 & 11,002 &, 000 \\
contente & 1437 & 8,45 & 2,505 & 1 & 10 & 5,237 & 10,140 &, 000 \\
frustrado & 1437 & 1,91 & 1,159 & 1 & 7 & 2,102 & 10,023 &, 000 \\
deprimido & 1437 & 1,80 & 1,076 & 1 & 10 & 2,207 & 10,803 &, 000 \\
com_raiva & 1437 & 2,11 & 1,321 & 1 & 10 & 3,035 & 8,477 &, 000 \\
irritado & 1437 & 2,06 & 1,302 & 1 & 10 & 2,955 & 8,377 &, 000 \\
furioso & 1437 & 2,01 & 1,192 & 1 & 10 & 2,624 & 8,647 &, 000 \\
triste & 1437 & 2,03 & 1,195 & 1 & 9 & 2,104 & 8,708 &, 000 \\
infeliz & 1437 & 2,17 & 1,256 & 1 & 9 & 1,965 & 8,072 &, 000 \\
AP01 & 1437 & 7,95 & 2,420 & 1 & 10 & 2,125 & 9,133 &, 000 \\
AP02 & 1437 & 9,04 & 1,389 & 1 & 10 & 1,613 & 9,582 &, 000 \\
AP03 & 1437 & 8,78 & 1,700 & 1 & 10 & 2,045 & 9,417 &, 000 \\
AP04 & 1437 & 8,43 & 1,958 & 1 & 10 & 2,343 & 8,183 &, 000 \\
RJ01 & 1437 & 2,05 & 1,424 & 1 & 10 & 2,784 & 8,803 &, 000 \\
RJ02 & 1437 & 1,92 & 1,305 & 1 & 10 & 2,621 & 9,960 &, 000 \\
RJ03 & 1437 & 1,44 & 1,376 & 1 & 9 & 2,700 & 9,011 &, 000 \\
RJ04 & 1437 & 1,76 & 1,739 & 1 & 10 & 2,151 & 8,685 &, 000 \\
\hline & & & & & & &
\end{tabular}

\subsection{Modelagem de equações estruturais}

Logo na primeira tentativa de modelagem dos dados, obtivemos indicadores satisfatórios. A AVE dos construtos foi superior a 0,50, a confiabilidade composta acima de 0,50 e a confiabilidade interna superior a 0,60 atendendo os parâmetros indicados pela literatura (CHIN, 1998; BIDO et al., 2011). Do mesmo modo, o índice de ajuste geral do modelo (Goodness of Fit - GoF) foi superior a 0,36, nível adequado para pesquisas em ciências sociais (BIDO et al., 2011). Os indicadores são apresentados na Figura 5.

\begin{tabular}{c|c|c|c|c|c}
\hline Dimensão & AVE & Confiabilidade Composta & $\mathbf{R}^{\mathbf{2}}$ & Cronbach & Redundância \\
\hline Aproximação & 0,517 & 0,809 & 0,305 & 0,689 & 0,134 \\
\hline Rejeição & 0,606 & 0,858 & 0,527 & 0,796 & 0,310 \\
\hline Emoções Negativas & 0,589 & 0,909 & 0,520 & 0,883 & 0,138 \\
\hline Emoções Positivas & 0,577 & 0,778 & 0,446 & 0,713 & $-0,006$ \\
\hline Layout & 0,566 & 0,838 & & 0,785 & \\
\hline Presença Social & 0,876 & 0,934 & & 0,860 & \\
\hline Atmosfera & 0,544 & 0,827 & & 0,723 & \\
\hline Teatralidade & 0,506 & 0,761 & & 0,617 & \\
\hline Médias & $0,576^{\star}$ & & 0,449 & & GoF $=0,508^{* *}$ \\
\hline
\end{tabular}

Figura $\mathbf{5}$ - Indicadores de ajuste do modelo

* Média ponderada pelo número de itens das dimensões.

** Média geométrica da média da AVE e do R2 médio. 
A validade convergente foi identificada por meio das cargas entre as variáveis manifestas e latentes $(>0,50)$ e a validade discriminante foi verificada por meio da comparação da raiz quadrada da AVE do construto e dos coeficientes de correlação entre o construto e as demais dimensões. Mesmo não havendo um limite mínimo para a diferença entre os indicadores ( $\mathrm{CHIN}$, 1998), é certo que a raiz quadrada da AVE não pode ser inferior à qualquer correlação. A Figura 6 apresenta os indicadores que comprovam a validade discriminante.

\begin{tabular}{c|c|c|c|c|c|c|c|c|c}
\hline Dimensão & AVE & $\mathbf{1}$ & $\mathbf{2}$ & $\mathbf{3}$ & $\mathbf{4}$ & $\mathbf{5}$ & $\mathbf{6}$ & $\mathbf{7}$ & $\mathbf{8}$ \\
\hline Aproximação & 0,517 & 0,719 & & & & & & \\
\hline Atmosfera & 0,606 & 0,609 & 0,778 & & & & & \\
\hline Emoções Negativas & 0,589 & $-0,525$ & $-0,598$ & 0,767 & & & & \\
\hline Emoções Positivas & 0,577 & 0,340 & 0,370 & $-0,341$ & 0,759 & & & \\
\hline Layout & 0,566 & 0,664 & 0,677 & $-0,658$ & 0,596 & 0,753 & & \\
\hline Presença Social & 0,876 & 0,118 & 0,096 & 0,093 & 0,392 & 0,175 & 0,936 & \\
\hline Rejeição & 0,544 & $-0,451$ & $-0,468$ & 0,723 & $-0,181$ & $-0,469$ & 0,261 & 0,738 & \\
\hline Teatralidade & 0,506 & 0,644 & 0,633 & $-0,482$ & 0,393 & 0,660 & 0,295 & $-0,399$ & 0,711 \\
\hline
\end{tabular}

Figura 6 - Validade discriminante do modelo

Nota: a diagonal em destaque representa a raiz quadrada da AVE da dimensão.

Por fim, a análise dos caminhos estruturais foi realizada, com utilização da técnica de reamostragem Bootstrap, para verificação das significâncias dos coeficientes. Nesta fase, foi possível realizar o teste das hipóteses. O resultado está apresentado na Figura 7.

\begin{tabular}{|c|c|c|c|c|c|c|c|c|}
\hline \multicolumn{2}{|c|}{$\begin{array}{c}\text { Hipótese / } \\
\text { Relação Esperada }\end{array}$} & \multirow{2}{*}{$\begin{array}{c}\text { Caminho } \\
\text { Layout -> Emoções Positivas }\end{array}$} & \multirow{2}{*}{$\begin{array}{c}\begin{array}{c}\text { Carga } \\
\text { original }\end{array} \\
0,613 \\
\end{array}$} & \multirow{2}{*}{$\begin{array}{c}\begin{array}{c}\text { Médias } \\
\text { Bootstrap }\end{array} \\
0,613 \\
\end{array}$} & \multirow{2}{*}{$\begin{array}{c}\text { Erro } \\
0,032 \\
\end{array}$} & \multirow{2}{*}{$\begin{array}{l}\text { Teste t } \\
19,046 \\
\end{array}$} & \multirow{2}{*}{ Sig } & \multirow{2}{*}{$\begin{array}{r}\text { Resultado } \\
\text { Aceita } \\
\end{array}$} \\
\hline $\mathrm{H} 1 \mathrm{a}$ & + & & & & & & & \\
\hline $\mathrm{H} 2 \mathrm{a}$ & + & Atmosfera -> Emoções Positivas & $-0,015$ & $-0,013$ & 0,037 & 0,410 & n.s. & Rejeitada \\
\hline $\mathrm{H} 2 \mathrm{~b}$ & - & Atmosfera -> Emoções Negativas & $-0,246$ & $-0,253$ & 0,049 & 5,033 & $\star * \star \star$ & Aceita \\
\hline $\mathrm{H} 3 \mathrm{a}$ & + & Teatralidade -> Emoções Positivas & 0,094 & $-0,096$ & 0,036 & 2,635 & $\star \star \star \star *$ & Aceita \\
\hline $\mathrm{H} 4 \mathrm{a}$ & + & Presença Social -> Emoções Positivas & 0,314 & 0,317 & 0,021 & 15,156 & $* * * *$ & Aceita \\
\hline $\mathrm{H} 4 \mathrm{~b}$ & - & Presença Social -> Emoções Negativas & 0,022 & 0,018 & 0,017 & 1,159 & n.s. & Rejeitada \\
\hline $\mathrm{H} 5 \mathrm{a}$ & + & Emoções Positivas -> Aproximação & 0,183 & 0,181 & 0,032 & 5,796 & $* * * *$ & Aceita \\
\hline $\mathrm{H} 5 \mathrm{~b}$ & - & Emoções Positivas -> Rejeição & $-0,074$ & 0,073 & 0,017 & 4,392 & $\star \star \star \star *$ & Aceita \\
\hline $\mathrm{H} 5 \mathrm{C}$ & + & Emoções Negativas -> Rejeição & 0,748 & 0,749 & 0,021 & 35,876 & $\star * * *$ & Aceita \\
\hline
\end{tabular}

Figura 7 - Significância dos caminhos e teste de hipóteses

Nota: os valores críticos de $t:{ }^{*} p<10 \%=1,645 ; * * p<5 \%=1,960 ;{ }^{* * *} p<1 \%=2,326$ e ****$p<0,1 \%=2,576$; n.s.=não significante.

\section{DISCUSSÃO DOS RESULTADOS}

Duas hipóteses, propostas na fase exploratória do estudo, não foram validades na análise dos dados empíricos. As hipóteses H2a (Haverá relação positiva entre a Atmosfera virtual da loja online e as emoções positivas do consumidor em relação ao varejista virtual) e H4b (Haverá relação negativa entre a Presença social virtual na loja online e as emoções negativas do 
consumidor em relação ao varejista virtual) não foram comprovadas.

Aparentemente, as cores de fundo e das fontes da webpage, assim como a qualidade sonora, não potencializam as emoções positivas em relação ao varejista online. Como houve a validação da hipótese H2b, que propôs a relação negativa entre as duas dimensões, é provável que a Atmosfera seja percebida somente quando atrapalhe a navegabilidade do consumidor.

Os achados suportam também o entendimento de que a Presença social virtual pode até contribuir para a formação das emoções positivas, porém sua falta não influencia as emoções negativas do consumidor. Com isto, é possível que o varejista que incorpore a Presença social virtual em seu website melhore sua avalição junto a seus consumidores aumentando sua diferenciação em um ambiente competitivo.

O modelo estrutural foi capaz de explicar 30,5\% da intenção de aproximação e 52,7\% da intenção de rejeição dos consumidores, indicando que as escalas propostas podem ser uma ferramenta de pesquisa interessante, tanto para acadêmicos quanto para os gestores.

O layout do website é muito mais relevante para a formação das emoções do consumidor quando comparado com as outras três dimensões analisadas. Reforça-se, com isto, a importância da construção de um cenário amigável, de fácil navegabilidade e com aparência estética moderna e agradável. Gestores desta área de negócios devem se preocupar em delegar o desenvolvimento de suas webpages para profissionais capacitados, como forma de minimizar o sentimento de rejeição de seu público alvo.

Em dissonância com resultados anteriores (VRECHOPOULOS et al.,2004), a dimensão Teatralidade virtual foi a que exerceu menor influência na formação das emoções, tanto negativas quanto positivas, dos consumidores online. Evidentemente, este resultado não se traduz como falta de importância dada pelos internautas para itens que compõem o construto, apenas indica que a variância da dimensão Teatralidade tem baixa relação com a variância das emoções. Como a grande maioria dos itens da dimensão Teatralidade teve média superior à 8 , bastante elevada para uma escala de 10 pontos, é possível que, por se tratar de websites nos quais os respondentes são habituados a comprar, a alta qualidade dos itens mensurados seja entendida como uma obrigação mínima do varejista virtual.

Identificamos também que as emoções negativas explicam mais as intenções de aproximação e rejeição quando comparadas com a influência das emoções positivas. Este fato remete à importância de se pesquisar o estado emocional dos consumidores. Os gestores devem focar na mensuração das emoções negativas e, ao primeiro sinal de seu surgimento ou incremento, erradicar seus antecedentes.

\subsection{Contribuições acadêmicas e limites desta pesquisa}

Por maiores que sejam suas limitações, acreditamos que este estudo proporciona alguma contribuição científica.

Inicialmente, o entendimento do Ambiente de loja online de maneira desagregada, por meio da análise de suas quatro dimensões formativas, é uma tentativa inicial de cobrir um gap da literatura. Neste sentido, recomenda-se que o modelo seja replicado em outros contextos e por meio de outros métodos de pesquisa (experimentais, por exemplo).

Outra contribuição é a identificação da força das emoções negativas na formação das intenções de aproximação e rejeição. A relação positiva entre as emoções positivas e as intenções comportamentais já é largamente apresentada na literatura (veja FARIAS \& SANTOS, 2000; ESPINOZA, 2004; LAROS \& STEENKAMP, 2005). 
Contudo, poucos estudos empíricos identificaram a maior relevância das emoções negativas como antecedente das intenções de aproximação e rejeição de consumidores, quer sejam presenciais quer sejam virtuais.

A característica não probabilística da amostra é um limite deste estudo, não permitindo que os resultados sejam extrapolados para o universo. Mesmo sendo uma limitação frequente em pesquisas da área de Administração, recomenda-se que, em futuros estudos, amostras aleatórias sejam utilizadas afim de conferir maior robustez aos resultados.

\subsection{Considerações finais}

O comércio online apresenta forte crescimento, tanto nos volumes negociados quanto na quantidade de consumidores que adotam esta ferramenta, despertando o interesse pelo entendimento de seus drivers de sucesso. Este estudo pretendeu contribuir, mesmo que minimamente, para o entendimento deste fenômeno.

\section{REFERÊNCIAS}

AUGER, P. The impact of interactivity and design sophistication on the performance of commercial websites for small businesses. Journal of Small Business Management, v. 4, n. 2, p. 119-137, 2005.

BABIN, B. J. et al. Modeling consumer satisfaction and word-of-mouth. The Journal of Services Marketing, v. 19, n. 3, p. 133-139, 2005.

BAKER, J.; LEVY, M.; GREWAL, D. An experimental approach to making retail store environmental decisions. Journal of Retailing, v. 68 n. 4, p. 445-460, 1992.

BAKER, J. et al. The influence of multiple store environment cues on perceived merchandise value and patronage intention. Journal of Marketing, v. 66, n. 2, p. 120-141, 2002.

BIDO, D. S. et al. Examinando a relação entre aprendizagem individual, grupal e organizacional em uma instituição financeira. REAd-Revista Eletrônica de Administração, v. 17, n. 1, p. 58-85, 2011.

BIERS, K.; RICHARDS, L. Color as a factor of product choice in e-commerce. The Review of Business Information Systems, v. 9, n. 4, p. 33-40, 2005.
BOSMANS, A. Scents and sensibility: when do (in)congruent ambient scents influence product evaluations?. Journal of Marketing, v. 70, n. 3, p. 3243, 2006.

BRANDÃO, M. M.; PARENTE, J. Brasileiro gosta de "muvuca"? Impacto da densidade humana no comportamento de compra. Revista de Administração de Empresas, v. 5, n. 6, p. 613-627, 2012.

CHIN, W. W. The partial least squares approach for structural equation modeling. In: Modern methods for business research. Nova York: Psychology Press, 1988.

CLARK, S.; BUCKINGHAM, S.; FORTIN, D. Investigating the impact of on-line store front atmospherics on perceived store personality. In: WILEY, J.; THIRKELL, P. (Eds.), Proceedings of the 2004 Australia New Zealand Marketing Academy Conference, Nova Zelândia, 2004

COSTA, F. C. X.; LARÁN, J. A. A compra por impulso em ambientes on-line. Revista de Administração de Empresas, v. 43, n. 4, p. 36-47, 2003.

COYLE, J.R.; THORSON, E. The effects of progressive levels of interactivity and vividness in web marketing sites. Journal of Advertising, v. 30, n. 3, p. 65-77, 2001. 


\section{REFERÊNCIAS}

CYR, D. et al. Beyond trust: web site design preferences across cultures. Journal of Global Information Management, v. 13, n. 4, p. 25-54, 2005.

DAILEY, L. Navigational web atmospherics: explaining the influence of restrictive navigation cues. Journal of Business Research, v. 57, n. 7, p. 795-803, 2004.

DEVELLIS, R. F. Scale development: Theory and applications (Book 26). Sage Publications, 2011.

DHOLAKIA, U. M. The usefulness of bidders' reputation ratings to sellers in online auctions. Journal of Interactive Marketing, v. 19, n. 1, p. 31-40, 2005.

EROGLU, S. A.; MACHLEIT, K. A.; DAVIS, L. M. Atmospheric qualities of online retailing: a conceptual model. Journal of Business Research, v. 54, n. 2, p. 177184,2001

Empirical testing of a model of online store atmospherics and shopper responses. Psychology \& Marketing, v. 20, n. 2, p. 139-150, 2003.

ESPINOZA, F. S. O impacto de experiências emocionais na atitude e intenção de comportamento do consumidor. 2004. Dissertação (Mestrado do Programa de Pósgraduação em Administração) - Universidade Federal do Rio Grande do Sul, Porto Alegre, 2004.

FARIAS, S. A. Atmosfera de loja on-line: o impacto do ambiente virtual na satisfação do consumidor e na atitude para com a compra. Revista de Administração, v. 42, n. 1, p. 31-41, 2007.

FARIAS, S.A.; SANTOS, R. C. Modelagem de equações estruturais e satisfação do consumidor. Revista de Administração Contemporânea, v. 4, n. 3, p. 107-132, 2000.
FIORE, A.M.; JIN, H.-J.; KIM, J. For fun and profit: hedonic value from image interactivity and responses toward an online store. Psychology \& Marketing, v. 22, n. 8, p. 669-694, 2005.

GARDNER, M. P. Mood states and consumer behavior: a critical review. Journal of Consumer Research, v. 12, n. 3, p. 281-300, 1985

GORN, G. J. et al. Waiting for the web: how screen color affects time perception. Journal of Marketing Research, v. 41, n. 2 , p. $215-25,2004$

GRIFFITH, D. A. An examination of the influences of store layout in online retailing. Journal of Business Research, v. 58, n. 10, p. 1391-1406, 2005.

GROEPPEL-KLEIN, A.; BAUN, D. The role of customers arousal for retail stores - results from an experimental pilot study using electrodermal activity as indicator. Advances in Consumer Research, v. 28, n. 1, p. 414419, 2001.

HAIR, J. F. et al. Análise multivariada de dados. Porto Alegre: Bookman, 2005.

HARDESTY, D. M.; CARLSON, J. P.; BEARDEN, W. Brand familiarity and invoice price effects on consumer evaluations: the moderating role of skepticism toward advertising. Journal of advertising, v. 31, n. 2, p. 1-15, 2002.

HSIAO, C.; SUN, B.; MORWITZ, V. G. The role of stated intentions in new product purchase forecasting. Advances in Econometrics, v. 16, p. 11-28, 2002.

KOERNIG, S. K. E-scapes: The electronic physical environment and service tangibility. Psychology \& Marketing, v. 20, n. 2, p. 151-167, 2003. 


\section{REFERÊNCIAS}

LAROS, F. J. IVI.; STEENKAMP, J. E. M. Emotions in consumer behavior: a hierarchical approach. Journal of Business Research, v. 58, n. 10, p. 1437-1445, 2005.

LEE, W.; BENBASAT, I. Designing an electronic commerce interface: attention and product memory as elicited by web design", Electronic Commerce Research and Applications, v. 2, n. 3, p. 240-53, 2003.

LEWISON, D. M. Retailing. 5. ed. Nova York: Macmillan College Publishing, 1994.

MALHOTRA, N. K. Pesquisa de marketing: uma orientação aplicada. Porto Alegre: Bookman, 2006.

MARTIN, B.A.S.; SHERRARD, M. J.; WENTZEL, D. The role of sensation seeking and need for cognition on web-site evaluations: a resource-matching perspective. Psychology \& Marketing, v. 22, n. 2, p. 109-26, 2005.

MCKINNEY, L. N. Creating a satisfying internet shopping experience via atmospheric variables. International Journal of Consumer Studies, v. 28, n. 3, p. 268-283, 2004.

MEHRABIAN, A.; RUSSELL, J. A. An approach to environmental psychology. Cambridge: MIT Press. 1974.

MUMMALANENI, V. An empirical investigation of web site characteristics, consumer emotional states and online shopping behaviors. Journal of Business Research, v. 58, n. 4, p. 526-532, 2005.

RICHINS, M. L. Measuring emotions in the consumption experience. Journal of Consumer Research, v. 24, n. 2, p. 127-142, 1997.

ROSEN, D. E.; PURINTON, E. Web site design: viewing the web as a cognitive landscape. Journal of Business Research, v. 57, n. 7, p. 787-94, 2004.
SHERMAN, E.; MATHUR, A.; SMITH, R. B. Store environment and consumer purchase behavior: mediating role of consumer emotions. Psychology \& Marketing, v. 14, n. 4, 361-378, 1997.

SONG, J.; ZAHEDI, F. A theoretical approach to web design in e-commerce: a belief reinforcement model. Management Science, v. 51, n. 8, p. 1219-1235, 2005.

SULLIVAN, M. the impact of pitch, volume and tempo on the atmospheric effects of music. International Journal of Retail and Distribution Management, v. 30, n. 6, p. 323-330, 2002.

SWANSON, S.R.; DAVIS, J C. The relationship of differential loci with perceived quality and behavioral intentions. The Journal of Services Marketing, v. 17, n. 2/3, p. 202-219, 2003.

TURLEY, L. W.; MILLIMAN, R. E. Atmospheric effects on shopping behavior: a review of the experimental evidence. Journal of Business Research, v. 49, n. 2, p. 193-211, 2000.

VRECHOPOULOS, A. P. et al. Virtual store layout: a comparison in the context of grocery retail. Journal of Retailing, v. 80, n. 1, p. 13-22, 2004.

WANG, L.; BAKER, J.; WAGNER, J. A. The role of atmospherics in e-tailing. The Proceedings of American Marketing Association, v. 13, p. 106-108, 2002.

WU, J. H.; YUAN, Y. Improving searching and reading performance: the effect of highlighting and text color coding. Information and Management, v. 40, n. 7, p. 617-37, 2003

YUN, Z.; GOOD, L. K. Developing customer loyalty from e-tail store image attributes. Managing Service Quality, v. 17, n. 1, p. 4-22, 2007. 